\title{
THE DYNAMICAL THEORY OF THE TIDES IN A POLAR BASIN
}

\author{
By G. R. Goldsbrougr. \\ [Read December 11th, 1913,-Received March 1st, 1914.]
}

\section{Introductory.}

THE problem here attacked is that of finding the tidal oscillations on a rotating globe in an ocean which is bounded by parallels of latitude. In this paper the case examined is that of a polar basin, so that only one boundary is to be considered. A similar analysis, however, can be applied to the double boundary problem-the zonal sea. In a further paper I intend to give the results of the latter case.

The only complete solution of a tidal problem hitherto found is that of Hough ${ }^{*}$ for the case of an ocean wholly covering a rotating globe. His results are in the form of series of spherical surface harmonics, and, in consequence, it is possible to include the effects of the self-attraction of the water particles. Poincare + and Love $\ddagger$ have suggested the use of the method of Ritz for the determination of tides in restricted basins; and Poincare $\S$ has also mentioned the possible application of Fredholm's integral equation method to these problems. Previously Kelvin !! had indicated a direct method of attacking the simpler problem of the tides in a zonal sea. Upon his method the following analysis is based. The form in which his results were left was such that it was almost impossible to make a numerical discussion of them; so that several alterations, suggested by Hough's work, have been made in the following paper. Since series of powers of cosines of the latitude are used, it is not possible to include the self-attraction. But the effects of the latter appear to be small, except in the vicinity of a coincidence of free and forced oscillations. The final results show that such an event cannot occur

\footnotetext{
* Phil. Trans., A, t. 189, p. 201, and t. 191, p. 139.

$\dagger$ Leçons de Mec. Cél., t. III, p. 297.

$\ddagger$ Proceedings of Wifth Inter. Congress of Math. (1912), Vol. II.

$\S$ Göttinger Vorträge (1910); Leçons, t. III, p. 233.

|| Phil. Mag., 1875.
} 
in basins of the dimensions considered; and hence the results without the self-attraction will represent well the complete expressions.

My thanks are due to Dr. T. H. Havelock for much useful advice and encouragement, and to Dr. Bromwich for many serviceable hints and comments.

\section{The Equations of Motion.}

Consider an ocean of uniform depth covering a rotating globe of negligible ellipticity, and bounded, if at all, by parallels of latitude. We shall principally discuss the case where the ocean includes one of the poles.

Let

$$
\begin{aligned}
& \theta=\text { co-latitude of a point on the surface; } \\
& \phi=\text { the longitude, reckoned eastward; } \\
& u=\text { the southward component of velocity; } \\
& v=\text { the eastward component of velocity; } \\
& \omega=\text { the angular velocity of the earth's rotation; } \\
& a=\text { the mean radius of the earth; } \\
& h=\text { the uniform depth of the ocean } \\
& \xi=\text { the tide beight, reckoned above the free surface; }
\end{aligned}
$$

and $\bar{\xi}=\frac{\Omega}{g}$, where $\Omega$ is the tide-producing potential.

Then, subject to certain approximations, the equations for the tidal oscillations are*

$$
\left.\begin{array}{l}
\frac{\partial u}{\partial t}-2 \omega v \cos \theta=-\frac{g}{a} \frac{\partial}{\partial \theta}(\xi-\bar{\xi}) \\
\frac{\partial v}{\partial t}+2 \omega u \cos \theta=-\frac{g}{a \sin \theta} \frac{\partial}{\partial \phi}(\xi-\bar{\xi}) \\
\frac{\partial \xi}{\partial t}=-\frac{h}{a \sin \theta}\left\{\frac{\partial}{\partial \theta}(u \sin \theta)+\frac{\partial v}{\partial \phi}\right\}
\end{array}\right\}
$$

$\bar{\xi}$ has the general form $H_{s} P_{2}^{s}(\cos \theta) \cos (\sigma t+s \phi+\epsilon)$, where $P_{2}^{s}$ is a spherical surface harmonic of rank $s$ and order 2 . For the three species of tides, $s$ takes the values $0,1,2$; and for each of these $\sigma$ has several values. $\dagger$

* Lamb, Hydrodyncmics, p. 314.

$\dagger$ Ibid, pp. 342, 343. For details as to the various values of $\sigma$, see Darwin, Scientific Papers, Vol. I, pp. 20-25. 
1913.] The pynamical theory of the tides in a polar basin.

Assume

$$
\begin{aligned}
& \xi \propto \cos (\sigma t+s \phi+\epsilon), \\
& u \propto \sin (\sigma t+s \phi+\epsilon), \\
& v \propto \cos (\sigma t+s \phi+\epsilon) .
\end{aligned}
$$

Substitute in equations (1) and solve for $u$ and $v$. On putting

$$
\frac{\sigma^{2}}{4 \omega^{2}}=f^{2}, \quad \frac{\omega^{2} a}{g}=m,
$$

we find $\quad u .4 m\left(f^{2}-1+\nu^{2}\right)=-\sigma \sqrt{ }\left(1-\nu^{2}\right) \frac{d \xi^{\prime}}{d \nu}-2 \cos \frac{\sqrt{ }\left(1-\nu^{2}\right) \xi^{\prime}}{\nu}$

$$
\left.\begin{array}{rl}
v .4 m\left(f^{2}-1+\nu^{2}\right) & =-2 \omega\left(1-\nu^{2}\right) \frac{d \xi^{\prime}}{d \nu}-\frac{\sigma s}{\nu} \xi^{\prime} \\
-\sigma \xi & =-\frac{h}{a \nu}\left[\sqrt{ }\left(1-\nu^{2}\right) \frac{d}{d \nu}(u . \nu)-s v\right]
\end{array}\right\},
$$

where we have changed the variable $\theta$ into $\nu,=\sin \theta$.

On eliminating $u$ and $v$, we have, finally, putting

$$
\begin{gathered}
\beta=\frac{4 \omega^{2} a^{2}}{g h}, \quad \xi^{\prime}=\xi-\bar{\xi} . \\
\beta \xi \nu=\sqrt{ }\left(1-\nu^{2}\right) \frac{d}{d \nu}\left[\frac{-\nu \sqrt{ }\left(1-\nu^{2}\right) \frac{d \xi^{\prime}}{d \nu}-\frac{s}{f} \sqrt{ }\left(1-\nu^{2}\right) \xi^{\prime}}{f^{2}-1+\nu^{2}}\right] \\
+\frac{\frac{s}{f}\left(1-\nu^{2}\right) \frac{d \xi^{\prime}}{d \nu}+\frac{s^{2}}{\nu} \xi^{\prime}}{f^{2}-1+\nu^{2}} .
\end{gathered}
$$

For the free oscillations, which we discuss first, $\bar{\xi}=0$, and $\xi^{\prime}$ is replaced by $\xi$.

If we suitably rearrange (3) for discussion, we find

$$
\begin{aligned}
& \nu\left(1-\nu^{2}\right) \frac{d^{2} \xi}{d \nu^{2}}-\frac{d \xi}{d \nu} {\left[2 \nu^{2}-1+\frac{2 \nu^{2}\left(1-\nu^{2}\right)}{f^{2}-1+\nu^{2}}\right] } \\
&-\xi\left[\frac{s}{f} \nu+\frac{s}{f} \frac{2 \nu\left(1-\nu^{2}\right)}{\left(f^{2}-1+\nu^{2}\right)}+\frac{s^{2}}{\nu}+\beta \nu\left(f^{2}-1+\nu^{2}\right)\right]=0 .
\end{aligned}
$$

The coefficients show singularities at

$$
\begin{aligned}
& \text { (i) } f^{2}-1+\nu^{2}=0 \text {, or } \nu= \pm \sqrt{1-f^{2}} \text {, } \\
& \text { (ii) } \quad 1-v^{2}=0 \text {, or } \nu= \pm 1 \text {, } \\
& \text { (iii) } \quad \nu=0 \text {, } \\
& \text { (iv) } \quad \nu=\infty \text {. }
\end{aligned}
$$

SER. 2. VOL. 14. NOO. 1223. 
With regard to (i), $f$ is unknown. It manifestly depends upon the depth of the sea and the extent of the basin. Moreover, for the same dimensions of the basin, $f$ has an infinity of values. It is impossible, therefore, to treat this singularity as the others. But, on examination, it appears that there is no singularity at all at the points $\nu \pm \sqrt{1-f^{2}}$. Or, rather, there is a special arrangement of the terms in the equation which prevents infinities occurring at these points.

When

$$
\begin{gathered}
f^{2}-1+\nu^{2}=0, \\
\frac{\sigma}{2 \omega}= \pm \cos \theta .
\end{gathered}
$$

If we take equations (1) and remove the time-and-longitude factor, the first pair present the form

$$
\left.\begin{array}{rl}
\sigma u-2 \omega v \cos \theta & =-\frac{g}{a} \frac{d \xi}{d \theta} \\
-\sigma v+2 \omega u \cos \theta & =\frac{g s}{a \sin \theta} \xi
\end{array}\right\} .
$$

On putting $\sigma=2 \omega \cos \theta$ in these, we find

$$
\begin{gathered}
\sigma(u-v)=-\frac{g}{a} \frac{d \xi}{d \theta}, \\
-\sigma(v-u)=\frac{g s}{a \sin \theta} \xi \\
\frac{d \xi}{d \theta}+\frac{s \xi}{\sin \theta}=0, \\
\sqrt{ }\left(1-\nu^{2}\right) \frac{d \xi}{d \nu}+\frac{s}{\nu} \xi=0 .
\end{gathered}
$$

That is,

or

Hence, when the denominators of the fractions in (3) vanish, the numerators do also; while the value of the fraction in such a case is a finite quantity-viz., $u$ in the first and $v$ in the second. Exactly the same happens when $\sigma=-2 \omega \cos \theta$. Hence this appearance of a singularity may be disregarded.*

Proceeding to (ii) we remark that, in the problem under consideration, $\nu$ is always positive. Hence, disregarding the singularity $\nu=-1$, we

* See Poincaré, Leçons de Méc. Cileste, t. I1, pp. 141, 142. The points $v= \pm \sqrt{ }\left(1-f^{2}\right)$ are, of course, "apparent singularities." 
proceed to find a solution of (4) valid in the vicinity $\nu=1$. For this purpose let

$$
\xi=(\nu-1)^{\alpha}\left[A_{0}+A_{1}(\nu-1)+A_{2}(\nu-1)^{2}+\ldots\right] \text {. }
$$

The indicial equation is

giving

$$
\begin{gathered}
\alpha(\alpha-1)+\frac{1}{2} \alpha=0, \\
\alpha=0, \frac{1}{2} .
\end{gathered}
$$

Hence the solutions suggested are of the character

$$
\xi=A_{0}+A_{1}(\nu-1)+A_{2}(\nu-1)^{2}+\ldots,
$$

and

$$
\xi=(\nu-1)^{\frac{1}{2}}\left\{B_{0}+B_{1}(\nu-1)+B_{2}(\nu-1)^{2}+\ldots\right\} \text {. }
$$

Usually the solutions are convergent in the vicinity of the singularity. But it is evident that, unless the coefficients in (7) bave special values, $\frac{d \xi}{d \nu}$ will have an infinity at $\nu=1$.

For case (iii) the indicial equation is

$$
\alpha(\alpha-1)+\alpha-s^{2}=0,
$$

whence

$$
\alpha= \pm s \text {. }
$$

The solutions indicated are then of the character

and

$$
\xi=\nu^{s} \quad\left\{A_{0}+A_{1} \nu+A_{2} \nu^{2}+\ldots ;,\right.
$$

In the case of the tides of first species (symmetrical oscillations) $s=0$. The indicial equation has then equal roots, $a=0$. The two solutions are then*

and

$$
\begin{aligned}
& \xi=\left[\nu^{\alpha}\left\{A_{0}+A_{1} \nu+A_{2} \nu^{2}+\ldots\right\}\right]_{\alpha=0}, \\
& \xi=\left[\frac{\partial}{\partial \alpha}\left\{\nu^{\alpha}\left(A_{0}+A_{1} \nu+A_{2} \nu^{2}+\ldots\right)\right\}\right]_{\alpha=0} .
\end{aligned}
$$

The latter may be written

$$
\begin{aligned}
\xi= & \log \nu\left[A_{0}+A_{1} \nu+A_{2} \nu^{2}+\ldots\right]_{a=0} \\
& +\left[\frac{\partial}{\partial \alpha}\left(A_{0}+A_{1} \nu+A_{2} \nu^{2}+\ldots\right)\right]_{a=0} .
\end{aligned}
$$

This solution, owing to the presence of the logarithm, has a singularity

* See Forsyth, Theory of Differential Equations, Tol. xv, p. 93. 
at $\nu=0$. The solution (9) likewise becomes infinite there.* Hence they are useless in considering a basin including the pole, though they would be of value in the case of a zonal sea. Solutions (6) and (7) are finite at $\nu=1$; so that it may be concluded that (8) is convergent from $\nu=0$ up to and including $\nu=1$. But, since from (7) $\frac{d \xi}{d \nu}$ probably has a singularity at $\nu=1$, we must investigate fully the convergence of the first derivative. $t$

Proceeding to find the relation between the coefficients in the series (8) from equation (3), it will be found advisable to break up the equation by the introduction of an auxiliary quantity $X$.

Let

$$
\nu \frac{d \xi^{\prime}}{d \nu}+\frac{s}{f} \xi^{\prime}=X\left(f^{2}-1+\nu^{2}\right)
$$

Then (3) becomes

$$
\beta f^{2} \nu^{2} \xi=-f^{2} \nu\left(1-\nu^{2}\right) \frac{d X}{d \nu}+f^{2} \nu^{2} X+s f\left(1-\nu^{2}\right) X+s^{2} \xi^{\prime}
$$

From the form of solution indicated in (8), we assume

and

$$
\begin{aligned}
\xi^{\prime} & =\sum_{0}^{\infty} A_{n} \nu^{s+n}, \\
\bar{\xi} & =\sum_{0}^{\infty} B_{n} \nu^{s+n}, \\
X & =\sum_{0}^{\infty} C_{n} \nu^{s+n} ;
\end{aligned}
$$

whence

$$
\xi=\zeta^{\prime}+\bar{\xi}=\sum_{0}^{\infty}\left(A_{n}+B_{n}\right) \nu^{s+n} .
$$

From (10), on substicuting and equating coefficients,

$$
A_{n}=\frac{\left(f^{2}-1\right) C_{n}+C_{n-2}}{n+s+s / f}
$$

and, from (11),

$$
\begin{aligned}
\beta f^{2}\left(A_{n-2}+B_{n-2}\right)-s^{2} A_{n}= & \left\{-f^{2}(n+s)+s f\right\} C_{n} \\
& +\left\{f^{2}(n+s-1)-s f\right\} C_{n-2} .
\end{aligned}
$$

* The treatment of this solution, since $s$ is an integer, would require to be carried out in a similar manner to the analogous solution of Bessel's equation. See Forsyth, Theory of Differential Equations, Vol. Iv, p. 101.

+ I am indebted to Dr. Bromwich for drawing my attention to this fact. 
It is simplest, though less convenient afterwards, to eliminate $A$ between (12) and (13). The result is the general relation

$$
\begin{aligned}
& -C_{n} \frac{n(2 s+n)}{n+s+s / f} \\
& +C_{n-2}\left\{-\frac{\beta\left(f^{2}-1\right)}{n-2+s+s / f}+\frac{(n+s)^{2}}{n+s+s / f}-1\right\} \\
& -C_{n-4} \frac{\beta}{n-2+s+s / f}=\beta . B_{n-2} .
\end{aligned}
$$

In (14) put $n=0$. Then

$$
0 . C_{0}=0 \text { or } C_{0} \text { is arbitrary. }
$$

Again, put $n=1$, and we find

$$
-C_{1} \frac{(2 s+1)}{1+s+s / f}=0, \quad \text { or } \quad C_{1}=0 .
$$

It follows that the series is one of even powers of $\nu$ only, factored by $\nu^{s}$; the odd coefficients all vanish. In discussing the solutions of (14) three cases, $s=0,1,2$, must be mentioned. For $s=0$ there exist

$$
B_{0}=\frac{2}{3} H_{0}, \quad \text { and } \quad B_{2}=-H_{0} \text {. }
$$

For $s=2$ there exists $B_{0}=H_{2}$ only.

In the other case, $s=1$, a different form must be given to the equations owing to the fact that the tide-producing potential is

$$
\bar{\xi}=H_{1} \nu \sqrt{ }\left(1-\nu^{2}\right) .
$$

This case is examined separately further on.

We will discuss (14), leaving $s$ general, though having more in mind the value $s=2$, as it is obvious that the simpler case $s=0$ can, by a slight readjustment of quantities, be made to fall within the same analysis.

Let

$$
\begin{gathered}
\left.L_{n}=\left\{\frac{\beta\left(f^{2}-1\right)}{n+s+s / f}-\frac{(n+2+s)^{2}}{n+2+s+s / f}+1\right\} \frac{n+2+s+s / f}{(n+2)(n+2+2 s)}\right\} \\
M_{n}=\frac{\beta(n+4+s+s / f)}{(n+4)(n+4+2 s)(n+2+s+s / f)} \\
\beta B_{0} \frac{2+s+s / f}{2(2+2 s)}=\kappa C_{0}
\end{gathered}
$$


Equations (14) then become

and thenceforward $\left.\begin{array}{rl}M_{0} C_{0}+L_{2} C_{2}+C_{4} & =0 \\ M_{n-2} C_{n-2}+L_{n} C_{n}+C_{n+2} & =0\end{array}\right\}$.

In equations (16), when $\kappa=0, f$ is unknown. When $f$ is given, $\kappa$, which determines $C_{0}$, is unknown. Hence they embrace both the free and the forced oscillations. In addition, the quantities $C$ must satisfy the boundary condition. If we suppose the basin we are dealing with bounded by a parallel of latitude $\nu=\nu_{1}$ we must have there

$$
u=0 \text {, }
$$

that is,

$$
\Sigma C_{n} \nu_{1}^{s+n}=0 . *
$$

When the ocean covers the whole globe, this equation is replaced by the condition that $\xi$ and $u$ are both finite when $\nu=1$.

Consider the convergence of the series defined by the last equation in (16). It is to be noted that we know nothing of the magnitude of $f$; it may even be negative. From (16) we have

$$
\begin{aligned}
\frac{C_{n+2}}{C_{n}}= & -L_{n}-\frac{M_{n-2}}{C_{n} / C_{n-2}} \\
= & \left\{-\frac{\beta\left(f^{2}-1\right)}{n+s+s / f}+\frac{(n+2+s)^{2}}{n+2+s+s / f}-1\right\} \frac{n+2+s+s / f}{(n+2)(n+2+2 s)} \\
& -\frac{\beta(n+2+s+s / f)}{(n+2)(n+2+2 s)(n+s+s / f)} \frac{1}{C_{n} / C_{n-2}} .
\end{aligned}
$$

It is obvious that in the limit $C_{n+2} / C_{n}$ tends either to unity or zero. More exactly, if $C_{n} / C_{n-2}$ is not a small quantity, whatever finite value $f$ may have, for values of $n$ sufficiently large, we may write

$$
C_{n+2} / C_{n}=1+\frac{0}{n}+\frac{\omega_{n}}{n^{2}},
$$

where $\omega_{n}$ is less than a fixed finite number.

Hence the series $\Sigma C_{n} \nu^{s+n}$ is only convergent so long as $|\nu|<1$.

* The quantity $X$ or $\Sigma C_{4 z^{s+n}}$ is a simple multiple of the velocity $u$, as is seen by reference to (2) and (10). 
Consequently, in order that the series may be valid at the point $\nu=1$, $f($ or $\kappa)$ in (16) nutust be so determined that

$$
\underset{n \rightarrow \infty}{L} \frac{C_{n+2}}{C_{n}}=0 .
$$

That is, $f$ (or $\kappa$ ) must be a solution of the convergent infinite continued fraction

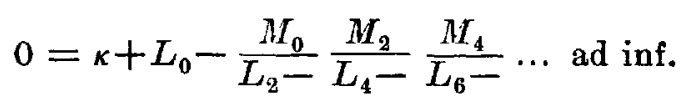

In this case the series $\Sigma C_{n} \nu^{n+8}$ is valid over the whole globe. But the application of (18) prevents the inclusion of any boundary condition.

If any other values of $f$ (or $\kappa$ ) than those given by (18) be used, $\frac{C_{n+2}}{C_{n}}$ tends to unity as $n$ increases. Hence the series $\Sigma C_{n} \nu^{9+n}$ is not valid at $\nu=1$. Consequently the polar basin for which our series give the tide heights cannot include the equator. For seas including the equator, but not the pole, series in powers of $\mu,=\sqrt{ }\left(1-\nu^{2}\right)$, are appropriate.

The series we have discussed is, of course, $\Sigma C_{n} \nu^{s+n}$, and not $\Sigma A_{n} \nu^{s+n}$, the former being used because of the simplicity of the relation between the coefficients. But there has, as yet, appeared no case in which the series was convergent and its derivative divergent at $\nu=1$, as was anticipated. Yet, if $\Sigma C_{n} \nu^{s+n}$ is absolutely convergent then, from (12), $\sum A_{n} \nu^{5+n}$ and its derivative are also convergent within the same circle of convergence. It may be that, owing to a lack of knowledge of the value of $f$, and a consequent lack of delicacy in the tests, or owing to the relation (12) masking some point, no indication of the expected result has appeared. One case, however, is interesting. For the luni-solar semi-diurnal tide $f$ is rigorously unity and $s=2$. Hence, from (12)

$$
A_{n}=\frac{C_{n-2}}{n+4}
$$

Also, from (14), $n C_{n}-\left(\frac{n^{2}+3 n}{n+4}\right) C_{n-2}+\frac{\beta}{n+2} C_{n-4}=0$,

or

$$
n(n+6) A_{n}-n(n+3) A_{n-2}+\beta A_{n-4}=0 .
$$

From this

$$
A_{n} / A_{n-2}=\frac{n+3}{n+6}-\frac{\beta}{n(n+6)} \frac{1}{A_{n-2} / A_{n-4}} .
$$


Hence, if $A_{n-2} / A_{n-4}$ is not small, the limiting form of $A_{n} / A_{n-2}$ is

$$
\frac{n+3}{n+6}, \text { or } \quad 1-\frac{3}{n} \text {. }
$$

This is identical with the limiting form of the ratio of the coefficients of $\nu^{n}$ and $\nu^{n-2}$ in the expansion of $\left(1-\nu^{2}\right)^{3}$. Hence the terms of the series $\Sigma A_{n} \nu^{n+8}$ become ultimately comparable with those of $(1-v)^{\frac{1}{2}}$, so that we may write

$$
\xi^{\prime}=L+M\left(1-y^{2}\right)^{2}
$$

where $L$ and $M$ are functions of $\nu$ that do not vanish when $\nu=1$. In this case clearly $\xi^{\prime}$ is finite at $z^{\prime}=1$, but $\frac{\partial \zeta^{\prime}}{\partial \nu}$ has an infinity there.*

The series are all undoubtedly convergent with their first derivatives so long as $\nu<1$. For the future we restrict ourselves to the condition that the basin shall not include nor extend up to the equator.

In seeking the solution for a polar basin we have to solve the equations

$$
\left.\begin{array}{r}
C_{0} \nu_{1}^{s}+C_{2} \nu_{1}^{s+2}+C_{4} \nu_{1}^{s+4}+\ldots=0 \\
\left(\kappa+L_{0}\right) C_{0}+C_{2}=0 \\
\ldots \quad \ldots \quad \ldots \quad \ldots \\
M_{n-2} C_{n-2}+L_{n} C_{n}+C_{n+2}=0
\end{array}\right) \cdot
$$

First consider a finite number of unknown quantities, say $(r+1)$. That is, suppose we can find a solution of the original differential equations by giving proper values to $C_{0}, C_{2}, \ldots, C_{2 r}$. If we suppose $f$ (or $\kappa$ ) known, we have only to express in terms of $C_{0}$ the remaining $r$ quantities $C$. For this purpose we require $r$ equations only, viz., the following :-

$$
\begin{aligned}
& C_{0} \nu_{1}^{s}+C_{2} \nu_{1}^{s+2}+C_{4} \nu_{1}^{s+4}+\ldots+C_{2, r y_{1}^{*+2 r}=0} \\
& \left(\kappa+L_{0}\right) C_{0}+C_{2}=0 \\
& M_{0} C_{0}+L_{2} C_{2}+C_{4}=0
\end{aligned}
$$

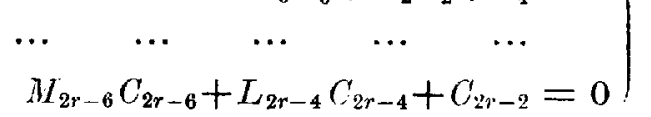


1913.] The dynamioal theory of the tides in a polar basin.

On solving these in the usual way, we have

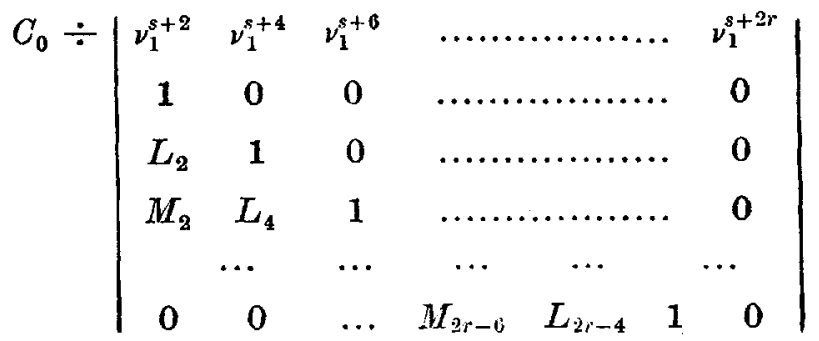

$$
\begin{aligned}
& =-C_{2} \div|\cdots| \\
& =C_{4} \div|\cdots| \\
& =\ldots
\end{aligned}
$$

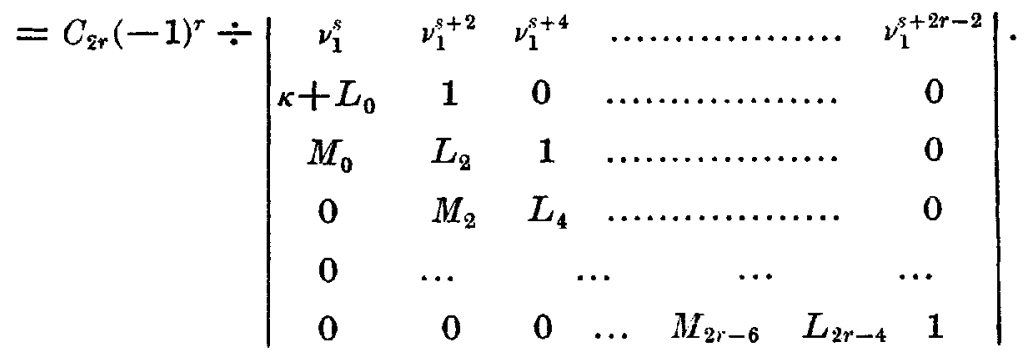

Hence, if we write the determinant associated with $C_{2 r}$ as $D_{2 r}$, we have

$$
(-1)^{r} . C_{2 r} \cdot \nu_{1}^{s+2 r}=C_{0} D_{2 r} \text {. }
$$

Now the original differential equations can only be satisfied by an infinite series, and, further, since the series $\Sigma C_{2 r} \nu_{1}^{s+2 r}$ converges, we have as a necessary consequence

$$
\underset{r \rightarrow \infty}{L} C_{2 r} \cdot v_{1}^{s+2 r}=0
$$

Hence we must have

$$
\underset{r \rightarrow \infty}{L} D_{2 r}=0 .
$$

This is the equation that determines the possible values of $f(o r k)$ for a given boundary. Written in extenso it is

$$
\left|\begin{array}{cccccc}
1 & \nu_{1}^{2} & \nu_{1}^{4} & \nu_{1}^{6} & \nu_{1}^{8} & \ldots \\
\kappa+L_{0} & 1 . & 0 & 0 & 0 & \ldots \\
M_{0} & L_{2} & 1 & 0 & 0 & \ldots \\
0 & M_{2} & L_{4} & 1 & 0 & \ldots \\
0 & 0 & M_{4} & L_{6} & 1 & \ldots \\
\ldots & \ldots & & \ldots & \ldots
\end{array}\right|=0 .
$$


In calculating the value of this determinant, we may use the sequence

$$
\begin{aligned}
& 0=1-\left(\kappa+L_{0}\right) \Delta_{2}-M_{0} \Delta_{4}, \\
& \Delta_{2}=\nu_{1}^{2}-L_{2} \Delta_{4}-M_{2} \Delta_{6} \text {, } \\
& \Delta_{n}=\nu_{1}^{\prime \prime}-L_{n} \Delta_{n+2}-M_{n} \Delta_{n+4} \text {; }
\end{aligned}
$$

where $\Delta_{n}$ represents the determinant beginning

$$
\left|\begin{array}{cccc}
\nu_{1}^{n} & \nu_{1}^{n+2} & \nu_{1}^{n+4} & \ldots \\
L_{n} & 1 & 0 & \ldots \\
M_{n} & L_{n+2} & 1 & \ldots \\
\ldots & \ldots & \ldots
\end{array}\right|
$$

In order that this determinant may be used at all we must show that, for a given finite value of $f$, it converges. The object will then be to find out for what value of $f$ it converges to zero.

From (15) it is easily seen that $\left|L_{n}\right|<1$ for sufficiently large values of $n$, and that $M_{n}$ is a small fraction. Hence

$$
\left|\Delta_{n}\right|<\nu^{n}\left|\begin{array}{cccc}
1 & \nu_{1}^{2} & \nu_{1}^{4} & \ldots \\
1 & 1 & 0 & \ldots \\
M_{n} & 1 & 1 & \ldots \\
0 & M_{n+2} & 1 & \ldots \\
\ldots & \ldots & & \ldots
\end{array}\right|
$$

if, on expanding, we replace the usual minus signs by plus. From this,

$$
\begin{aligned}
& \frac{\left|\Delta_{n}\right|}{\nu_{1}^{n}}<1+\nu_{1}^{2}+\nu_{1}^{4}\left|\begin{array}{cc}
1 & 1 \\
M_{n} & 1
\end{array}\right|+\nu_{1}^{i}\left|\begin{array}{ccc}
1 & 1 & 0 \\
M_{n} & 1 & 1 \\
0 & M_{n+2} & 1
\end{array}\right| \\
& +\nu_{1}^{8}\left|\begin{array}{cccc}
1 & 1 & 0 & 0 \\
M_{n} & 1 & 1 & 0 \\
0 & M_{n+2} & 1 & 1 \\
0 & 0 & M_{n+4} & 1
\end{array}\right|+\ldots
\end{aligned}
$$




$$
\text { Or, } \quad \begin{aligned}
\frac{\left|\Delta_{n}\right|}{\nu_{1}^{n}}<1+\nu_{1}^{2} & +\nu_{1}^{4}\left(1+M_{n}\right)+\nu_{1}^{6}\left(1+M_{n}+M_{n+2}\right) \\
& +\nu_{1}^{8}\left(1+M_{n}+M_{n+2}+M_{n+4}+M_{n} M_{n+4}\right) \\
& +\ldots \\
<1+\nu_{1}^{2} & +\nu_{1}^{4}\left(1+M_{n}\right) \\
& +\nu_{1}^{6}\left(1+M_{n}\right)\left(1+M_{n+2}\right) \\
& +\nu_{1}^{8}\left(1+M_{n}\right)\left(1+M_{n+2}\right)\left(1+M_{n+4}\right) \\
& +\ldots
\end{aligned}
$$

The ratio of the $(p+1)$-th term to the $p$-th of this last series is

$$
\text { i.e., } \quad \nu_{1}^{2}\left\{1+\frac{\beta(n+2 p-2+s+s / f)}{(n+2 p+2)(n+2 p-2+2 s)(n+2 p-4+s+s / f)}\right\},
$$

i.e., $<1$, after some fixed value of $s$, since $\nu<1$.

Hence $\frac{\left|\Delta_{n}\right|}{y_{1}^{n}}$ is less than the sum of an absolutely convergent series, and so has a finite value. It follows at once that the original determinant on the left-hand side of (21) has a finite value for finite values of $f$.

Again, the series in (23) decreases in sum as $n$ increases, since each coefficient decreases. The limit is $\frac{1}{1-\nu_{1}^{2}}$. Also the factor $\nu_{1}^{n}$ decreases to zero as $n$ increases. Hence the series $\Delta_{2}, \Delta_{4}, \ldots, \Delta_{2 r}$ ultimately decreases to zero in the manner of a geometrical progression.

In the case of the tides of the second species $(s=1)$, the form of the tide-producing potential suggests the substitution

$$
\begin{aligned}
\xi^{\prime} & =\sqrt{ }\left(1-\nu^{2}\right) z^{\prime}, \\
\bar{\xi} & =\sqrt{ }\left(1-\nu^{2}\right) \bar{z}, \\
\xi & =\sqrt{ }\left(1-\nu^{2}\right) z .
\end{aligned}
$$

Equations (2) then give, for the velocities,

$$
\begin{aligned}
& u .4 m\left(f^{2}-1+\nu^{2}\right)=-\sigma\left\{\left(1-\nu^{2}\right) \frac{d z^{\prime}}{d \nu}-\nu z^{\prime}+\frac{2 \omega}{\sigma}\left(1-\nu^{2}\right) \frac{z^{\prime}}{\nu}\right\}, \\
& v .4 m\left(f^{2}-1+\nu^{2}\right)=-\sqrt{ }\left(1-\nu^{2}\right)\left\{2 \omega\left(1-\nu^{2}\right) \frac{d z^{\prime}}{d \nu}-2 \omega \nu z^{\prime}+\sigma \frac{z^{\prime}}{\nu}\right\} .
\end{aligned}
$$


And in place of (3) we have

$$
\begin{aligned}
\beta z . \nu= & -\frac{d}{d \nu}\left[\frac{\nu\left(1-\nu^{2}\right) \frac{d z^{\prime}}{d \nu}-\nu^{2} z^{\prime}+\frac{1-\nu^{2}}{f} z^{\prime}}{f^{2}-1+\nu^{2}}\right] \\
& +\frac{\frac{1-\nu^{2}}{f} \frac{d z^{\prime}}{d \nu}-\frac{\nu z^{\prime}}{f}+\frac{z^{\prime}}{\nu}}{f^{2}-1+\nu^{2}} .
\end{aligned}
$$

The coefficients in (25) have singularities at $\nu=0$ and $\nu= \pm 1$. The indicial equation corresponding to a solution in the vicinity of $\nu=0$, is

$$
-a(a-1)-a+1=0 ;
$$

whence

$$
\alpha= \pm 1 \text {. }
$$

Corresponding to $\nu=+1$ ( $\nu=-1$ cannot appear in the problem), we have

whence

$$
2 \alpha(\alpha-1)+3 \alpha=0 \text {; }
$$$$
a=0,-\frac{1}{2} \text {. }
$$

These results might have been inferred, of course, from the results found previously. It seems probable that a discontinuity in the series will occur at $\nu=1$.

$$
\text { Let } \quad \nu\left(1-\nu^{2}\right) \frac{d z^{\prime}}{d \nu^{\prime}}-\nu^{2} z^{\prime}+\frac{1-\nu^{2}}{f} z^{\prime}=X\left(f^{2}-1+\nu^{2}\right) \text {. }
$$

Equation (25) then becomes

$$
f^{2} \beta z \nu^{2}=-\frac{d X}{d \nu} f^{2} \nu+f X+z^{\prime} .
$$

As before, assume as a solution in the vicinity of $\nu=0$,

$$
\begin{aligned}
& z^{\prime}=\sum_{1}^{\infty} A_{n} \nu^{n}, \\
& \bar{z}=\sum_{1}^{\infty} B_{n} \nu^{n}, \\
& X=\sum_{1} C_{n} \nu^{n} .
\end{aligned}
$$

and

On substituting and equating coefficients, (28) gives

$$
C_{n}\left(f-f^{2} n\right)=f^{3} \beta . A_{n-2}-A_{n}+f^{2} \beta . B_{n-2} .
$$


1913.] The dynamical theory of the tides in a polar basin.

Similarly (27) gives

$$
A_{n}\left(n+\frac{1}{f}\right)-A_{n-2}\left(n-1+\frac{1}{f}\right)=\left(f^{2}-1\right) C_{n}+C_{n-2} .
$$

Whence, on eliminating $C$ between these two equations and reducing, the result is

$$
\begin{gathered}
A_{n}+A_{n-2}\left\{\frac{\left(f^{2}-1\right) \beta}{\frac{1}{f}-n}-\frac{\left.(n-2)(n-1)-\frac{1}{f}\right)}{\frac{1}{f}-(n-2)}\right\} \frac{1}{n^{2}-1} \\
+A_{n-4} \frac{\beta}{\frac{1}{f}-(n-2)} \frac{\frac{1}{f}-n}{n^{2}-1} \\
=-\frac{\left(f^{2}-1\right) \beta}{n^{2}-1} B_{n-2}-\frac{\left(\frac{1}{f}-n\right) \beta}{\left(n^{2}-1\right)\left(\frac{1}{f}-(n-2)\right)} B_{n-4} .
\end{gathered}
$$

The convergence of the series determined by (29) is discussed in the same manner as before.

$$
\begin{aligned}
A_{n} / A_{n-2}= & \left\{-\frac{\left(f^{2}-1\right) \beta}{n-\frac{1}{f}}+\frac{(n-2)(n-1)-\frac{1}{f}}{(n-2)-\frac{1}{f}}\right\} \frac{n-\frac{1}{f}}{n^{2}-1} \\
& -\frac{\beta\left(n-\frac{1}{f}\right)}{\left(n+2-\frac{1}{f}\right)\left(n^{2}-1\right)} \frac{1}{A_{n-2} / A_{n-4}} .
\end{aligned}
$$

It is possible, as in the last case, for $A_{n} / A_{n-2}$ to converge to zero. In the other case, when $A_{n-2} / A_{n-4}$ is not small, for sufficiently large values of $n$,

$$
A_{n} / A_{n-2}=1-\frac{1}{n}+\frac{\omega_{n}}{n^{2}} \text {. }
$$

Hence the series $\Sigma A_{n} \nu^{n}$ is convergent only so long as $|\nu|<1$.

Equation (18) gives one type of free oscillations for the case where the ocean covers the whole globe; but, as is well known, * there are two types, 
characterized by symmetry or asymmetry with regard to the equator. Let us find an equation for the other set.

The original differential equations (2) and (3) may be satisfied by a solution of the form

$$
\xi^{\prime}=\sqrt{ }\left(1-\nu^{2}\right) z^{\prime}, \ldots,
$$

where $z^{\prime}$ is a series of powers of $\nu$.

On making such a substitution, we find

$$
\begin{aligned}
u .4 m\left(f^{2}-1+\nu^{2}\right)= & -\sigma\left\{\left(1-\nu^{2}\right) \frac{d z^{\prime}}{d \nu}-\nu z^{\prime}\right\}-2 \omega s\left(1-\nu^{2}\right) \frac{z^{\prime}}{\nu} \\
v .4 m\left(f^{2}-1+\nu^{2}\right)= & -2 \omega\left\{\left(1-\nu^{2}\right)^{\frac{3}{2}} \frac{d z^{\prime}}{d \nu}-\nu \sqrt{ }\left(1-\nu^{2}\right) z^{\prime}\right\}-\frac{\sigma s}{\nu} \sqrt{ }\left(1-\nu^{2}\right) z^{\prime} \\
\beta z \nu= & -\frac{d}{d \nu}\left[\frac{\nu\left(1-\nu^{2}\right) \frac{d z^{\prime}}{d \nu}-\nu^{2} z^{\prime}+\left(1-\nu^{2}\right) \frac{s}{f} z^{\prime}}{f^{2}-1+\nu^{2}}\right] \\
& +\frac{\frac{s}{f}\left(1-\nu^{2}\right) \frac{d z^{\prime}}{d \nu}-\frac{s}{f} \nu z^{\prime}+\frac{s^{2}}{\nu} z^{\prime}}{f^{2}-1+\nu^{2}}
\end{aligned}
$$

The equation (18a) has apparent singularities at $\nu= \pm \sqrt{ }\left(1-f^{2}\right)$, and real singularities at $\nu= \pm 1, \nu=0$.

At $\nu=0$, the indices are $\pm s$.

Hence we may expect to find a solution in the vicinity of $\nu=0$, of the type

$$
z^{\prime}=\sum_{n=0}^{\infty} A_{n} \nu^{s+n}
$$

The solution involving the second index, $-s$, includes a logarithm, and hence is infinite at the pole.

On substituting the value $(18 b)$ in $(18 a)$ in the same manner as was done previously we arrive at the relation, for free oscillations,

$$
\begin{gathered}
A_{n} \frac{n^{2}+2 n s}{n+s-s / f}+A_{n-2}\left\{\frac{\beta\left(f^{2}-1\right)}{n+s-s / f}-\frac{(n+s-1)(n+s-2)-s / f}{n-2+s-s / f}\right\} \\
+A_{n-4} \frac{\beta}{n-2+s-s / f}=0 .
\end{gathered}
$$

Equation (29) is included in this as a special case $(s=1)$. The corresponding alternative for the tides of the second species is found by putting $s=1$ in (14). 
Equation (18c) differs but little in form from (14); but it is easy to verify that it provides the oscillations omitted in (14). In the case of no rotation, the frequency is given by $\frac{\sigma^{2} a^{2}}{g h}=n(n+1)$, where $n$ may take any integral value.

Putting $\omega=0$ in (18c) and (14), it is obvious that the frequencies are given by

$$
\frac{\sigma^{2} a^{2}}{g h}=(n+s)(n+s-1)
$$

in the first case, and by $\frac{\sigma^{2} a^{2}}{g h}=(n+s-1)(n+s-2)$

in the second case ; where $s=0$ or 2 , and $n$ is any even integer.* These two statements each supply the other's deficit and complete the whole series of frequencies.

Hence (18c), with a corresponding boundary condition, will give a new set of free oscillations in the same manner as those already indicated. The series defined by $(18 c)$ is subject to the same limitations as that defined by (14), and hence the free oscillations for the ocean covering the whole globe will be given by an infinite continued fraction of the same type as (18), and for which the quantities $L$ and $M$ are given in (18c). $\mathrm{As}$, however, these types of motion have no relation to those excited by the tide-producing potential, their interest is much diminished, and I have not proposed to pay them any further attention.

If equation (18a) be taken and reduced so as to express oscillations in a plane circular sea, by putting $\nu=x / a$ and making $a$ infinite, we find

$$
\frac{1}{x} \frac{d}{d x}\left[x \frac{d z^{\prime}}{d x}\right]+\left\{\left(f^{2}-1\right) \frac{4 \omega^{2}}{g h}-\frac{s^{2}}{x^{2}}\right\} z^{\prime}=-\left(f^{2}-1\right) \frac{4 \omega^{2}}{g h} \bar{z},
$$

which is the familiar form of the equation. It is noteworthy that (3) reduces to precisely the same form. The two sets of oscillations apparently merge into one another as the basin narrows, and finally present only one solution, of the form

$$
z=J_{\star}(\kappa x), \quad \kappa^{2}=\left(f^{2}-1\right) \frac{4 \omega^{2}}{g h} .
$$

* The circumstance $s=0, n=2$ in (14) is discussed later. 
The two classes of oscillations in the general case have the forms

$$
\sum_{n=0}^{\infty} A_{n} \nu^{n+s}
$$

and

$$
\sqrt{ }\left(1-\nu^{2}\right) \sum_{n=0}^{\infty} B_{n} \nu^{n+s}
$$

Since each of the series converges at the equator $(\nu=1)$, the tide height for the second set of oscillations will vanish there, while that of the first will not. This affords a distinction between the two types when expressed in terms of $\nu$.

\section{Tides of the First Species.}

The tides of the first species are determined from the previous formulæ by putting $s=0$. From (14) we have

$$
n C_{n}+C_{n-2}\left\{\frac{\beta\left(f^{2}-1\right)}{n-2}-(n-1)\right\}+C_{n-4} \frac{\beta}{n-2}=-\beta . B_{n-2}
$$

together with

$$
A_{n}=\frac{\left(f^{2}-1\right) C_{n}+C_{n-2}}{n}
$$

These equations are not satisfactory when $n=0$ or 2 . From the first when $n=0$, we have

$$
0 . C_{0}=0 \text {, or } C_{0} \text { is arbitrary. }
$$

But, from (31),

$$
A_{0}=\frac{\left(f^{2}-1\right) C_{0}}{0},
$$

an infinite quantity.

In place of the arbitrary $C_{0}$, choose $A_{0}$, which is such that

$$
n A_{n}=\left(f^{2}-1\right) C_{n} \text {, when } n \text { is zero. }
$$

Then, from (30), when $n=2$,

$$
2 C_{2}+\beta A_{0}=0, \text { or }-\beta B_{0},
$$

according as the oscillations are free or forced. 
1913.] The dynamical theory of the tides in a polar basin.

The relation (32) requires that $C_{0}$ should be zero. Hence, when $n=4$, the condition is

$$
4 C_{4}+C_{2}\left(\frac{\beta\left(f^{2}-1\right)}{2}-3\right)=0, \text { or }-\beta B_{2} .
$$

After these values of $n$, the relation (30) holds good. The boundary condition is simply $\Sigma C_{n} \nu_{1}^{n}=0$.

The determinant for free oscillations is

where

$$
\left|\begin{array}{ccccccc}
1 & \nu_{1}^{2} & \nu_{1}^{4} & \nu_{1}^{6} & \nu_{1}^{8} & \ldots & \text { ad inf. } \\
L_{2} & 1 & 0 & 0 & 0 & \ldots & \\
M_{2} & L_{4} & 1 & 0 & 0 & \ldots & \\
0 & M_{4} & L_{6} & 1 & 0 & \ldots & \\
0 & 0 & M_{6} & L_{8} & 1 & \ldots & \\
\ldots & & \ldots & \ldots & \ldots & \ldots
\end{array}\right|=0
$$

$$
\begin{aligned}
& L_{n}=\frac{\beta\left(f^{2}-1\right)}{n(n+2)}-\frac{n+1}{n+2}, \\
& M_{n}=\frac{\beta}{(n+2)(n+4)} .
\end{aligned}
$$

In attempting to find the roots of this equation we may take as a rough approximation to the first root the value given by putting all the powers of $\nu_{1}$ above the second equal to zero. That is

$$
L_{2}=\frac{1}{\nu_{1}^{2}}
$$

Another approximation may be obtained from the original differential equation (3).

Putting $s=0$, it becomes

$$
\frac{\sqrt{ }\left(1-\nu^{2}\right)}{\nu} \frac{d}{d \nu}\left[\frac{\nu \sqrt{ }\left(1-\nu^{2}\right)}{f^{2}-1+\nu^{2}} \frac{d \xi}{d \nu}\right]+\beta \xi=0 .
$$

If we put $\nu=x / a$, and then make $a$ indefinitely great, the equation becomes

$$
\frac{1}{x} \frac{d}{d x}\left(x \frac{d \xi}{d x}\right)+\left(f^{2}-1\right) \frac{4 \omega^{2}}{g h} \xi=0 .
$$

SER. 2. VOL. 14. NO. 1224. 
The solution of this is

$$
\xi=J_{0}\left\{x \sqrt{\frac{\left(f^{2}-1\right) 4 \omega^{2}}{g h}}\right\} .
$$

The boundary condition then becomes

$$
J_{0}^{\prime}\left\{v_{1} \sqrt{\left(f^{2}-1\right) \frac{4 \omega^{2} a^{2}}{g h}}\right\}=-J_{1}\left\{v_{1} \sqrt{\left(f^{2}-1\right) \beta}\right\}=0 .
$$

Equation (37) can readily be solved, and the values of $f$ so found used as first approximations to the solution for the lowest roots of (35).

This problem has been carried to a second approximation by Proudman.*

Having determined a value for $f$, the determinant can then be evaluated by use of the sequence

$$
\Delta_{2 r}=\nu_{1}^{2 r-2}-L_{2 r} \Delta_{2 r+2}-M_{2 r} \Delta_{2 r+4} .
$$

The quantity $\Delta_{2 r}$ eventually becomes equal to $\nu_{1}^{2 r-2}$ in value. Hence, for a sufficiently high value of $r$, we may reject $\Delta_{2 r+2}, \Delta_{2 r+4}, \ldots$, \&c., and putting $\Delta_{2 r}=\nu_{1}^{2 r-2}$, evaluate in succession $\Delta_{2 r-2}, \Delta_{2 r-4}, \ldots, \Delta_{2}$.

The value of $\Delta_{2}$ will not be zero. A second and modified value of $f$ is then chosen, and the value of $\Delta_{2}$ again calculated. By this process of trial and error and final interpolation a value of $f$ may be found that satisfies (35).

Since $\beta=\frac{4 \omega^{2} a^{2}}{g h}$ and $f=\frac{\sigma}{2 \omega}$, when $\omega=0, L_{n}$ becomes

$$
\frac{\frac{a^{2}}{g h} \sigma^{2}}{n(n+2)}-\frac{n+1}{n+2}
$$

and $M_{n}$ becomes zero.

Hence, by a very slight modification of the preceding process, the frequencies for the case of no rotation may be calculated.

Following out this process the results given below were obtained. The sequences used in calculating the value of the determinant (35), as they ultimately become the geometric progression $\Sigma_{\nu_{1}}^{2 r}$, converge rapidly for small values of $\nu_{1}$. But as $\nu_{1}$ approaches unity the work becomes exceedingly laborious. 
TABLE 1.-Lowest frequencies of tides of first species.

Boundary $\nu_{1}=\frac{1}{4}$, Lat. $75^{\circ} 30^{\prime}$.

\begin{tabular}{|c|c|c|c|}
\hline Depth. & $\begin{array}{c}\text { Value of } \\
f^{2}=\sigma^{2} / 4 \omega^{2}\end{array}$ & $\begin{array}{c}\text { Period in } \\
\text { sidereal time. }\end{array}$ & $\begin{array}{c}\text { Period in case of } \\
\text { no rotatiou. }\end{array}$ \\
\hline$\left\{\begin{array}{l}58,080 \text { feet } \\
\beta=5\end{array}\right.$ & $45 \cdot 08$ & $1 \mathrm{~h} .47 \mathrm{~m}$. & $1 \mathrm{~h} .48 \mathrm{~m}$. \\
\hline$\left\{\begin{array}{l}29,040 \\
\beta=10\end{array} "\right.$ & $23 \cdot 05$ & $2 \mathrm{~h} .30 \mathrm{~m}$. & $2 \mathrm{~h} .33 \mathrm{~m}$. \\
\hline$\left\{\begin{array}{l}14,520 \\
\beta=20\end{array}\right.$, & $12 \cdot 02$ & $3 \mathrm{~h} .27 \mathrm{~m}$. & $3 \mathrm{~h} .37 \mathrm{~m}$. \\
\hline$\left\{\begin{array}{l}7,260 \\
\beta=40\end{array} "\right.$ & 6.51 & $4 \mathrm{~h} .42 \mathrm{~m}$. & $5 \mathrm{~h} . \quad 6 \mathrm{~m}$. \\
\hline
\end{tabular}

TABLE II.-Lowest frequencies of tides of first species.

Boundary $\nu_{1}=\frac{1}{2}$, Lat. $60^{\circ}$.

\begin{tabular}{|c|c|c|c|}
\hline Depth. & $\begin{array}{c}\begin{array}{c}\text { Value of } \\
f^{2}=\sigma^{2} / 4 \omega^{2}\end{array}\end{array}$ & $\begin{array}{l}\text { Period in } \\
\text { sidereal time. }\end{array}$ & $\begin{array}{c}\text { Period in case of } \\
\text { no rotation. }\end{array}$ \\
\hline$\left\{\begin{array}{l}58,080 \text { feet } \\
\beta=5\end{array}\right.$ & $11 \cdot 74$ & $3 \mathrm{~h} .80 \mathrm{~m}$. & $3 \mathrm{~h} .40 \mathrm{~m}$. \\
\hline$\left\{\begin{array}{l}29,040 \\
\beta=10\end{array}\right.$ & $6 \cdot 28$ & $4 \mathrm{~h} .47 \mathrm{~m}$. & $5 \mathrm{~h} .12 \mathrm{~m}$. \\
\hline$\left\{\begin{array}{l}14,520 \\
\beta=20\end{array} "\right.$ & $3 \cdot 59$ & $6 \mathrm{~h} .20 \mathrm{~m}$. & $7 \mathrm{~h} .22 \mathrm{~m}$. \\
\hline$\left\{\begin{array}{l}7,260 \\
\beta=40\end{array}\right)$ & $2 \cdot 25$ & $8 \mathrm{~h} . \quad 0 \mathrm{~m}$. & $10 \mathrm{~h} .40 \mathrm{~m}$. \\
\hline
\end{tabular}

The principal forced tide of the first species is the lunar-fortnightly. For this the tide-producing potential is $H_{0}\left(\cos ^{2} \theta-\frac{1}{3}\right)$ or $H_{0}\left(\frac{2}{3}-\nu^{2}\right)$, and the frequency is $f^{2}=\cdot 00133$. We have, therefore, $B_{0}=\frac{2}{3} H_{0}$ and 
$B_{2}=-H_{0}$. Hence, putting $H_{0}={ }_{\kappa} C_{2}$, we have to solve the equations

$$
\left.\begin{array}{c}
\sum_{n=0}^{n=\infty} C_{2 n} \nu_{1}^{2 n}=0 \\
C_{2}+\frac{\beta}{2} A_{0}=-\frac{\beta}{3} H_{0} \\
\left(L_{2}-\frac{\beta_{\kappa}}{4}\right) C_{2}+C_{4}=0 \\
M_{n-4} C_{n-4}+L_{n-2} C_{n-2}+C_{n}=0
\end{array}\right\} .
$$

and thereafter

In the first of these equations it is to be remembered that, according to our previous arrangement, $C_{0}=0$.

Leaving for a moment the first pair of equations (38), the last set can be solved. We have

$$
\begin{aligned}
C_{2}=-C_{4} \div D_{2} & =C_{6} \div D_{4}=-C_{8} \div D_{6} \\
& =\ldots \\
& =(-1)^{r-1} C_{2 r} \div D_{2 r-2} \\
& =\ldots ;
\end{aligned}
$$

where we have written $D_{2}$ in place of

$$
\left|\begin{array}{cccccccc}
L_{2}-\frac{1}{4} \beta \kappa & 1 & 0 & 0 & 0 & \ldots & 0 & 0 \\
M_{2} & L_{4} & 1 & 0 & 0 & \ldots & 0 & 0 \\
0 & M_{4} & L_{6} & 1 & 0 & \ldots & 0 & 0 \\
0 & 0 & M_{6} & L_{8} & 1 & \ldots & 0 & 0 \\
\ldots & \ldots & \ldots & \ldots & \ldots & \ldots & \ldots & \ldots \\
0 & 0 & 0 & 0 & 0 & \ldots & M_{2 r-2} & L_{2 r}
\end{array}\right|
$$

The quantities $D$ are related by the succession

$$
D_{2 r}=L_{2 r} D_{2 r-2}-M_{3 r-2} D_{2 r-4} \text {. }
$$

The method of calculation is the following. The quantities $D$ are calculated in succession by means of (40), the quantity $\kappa$ being left undetermined. These are then substituted in the boundary equation and a linear equation determining $\alpha$ results. From the condition 
$C_{2}=H_{0} / \kappa, C_{2}$ is found. All the coefficients $C$ then follow. Finally, $A_{0}$ is determined from the second equation of (38), and the remaining $A^{\prime}$ 's from (31).

The tide height is then

$$
\xi=H_{0}\left(\frac{2}{3}-\nu^{2}\right)+A_{0}+\sum_{n=2}^{n=\infty}\left[\frac{\left(f^{2}-1\right) C_{n}+C_{n-2}}{n}\right] \nu^{n} .
$$

The calculation is possible for any value of $\nu_{1}$ less than unity; but, of course, it is very tedious owing to the slow convergence when $\nu_{1}$ is near unity.

The following series for the tide heights were calculated with fourfigure logarithms. Only three significant figures have been retained in the results, as, owing to the complexity of the processes, much doubt attaches to the fourth significant figure.

Lunar fortnightly tide. Bo: $n$ dary $\nu_{1}=\frac{1}{4}$, Lat. $75^{\circ} 30^{\prime}$.

Depth 58,080 feet ; $\beta=5$.

$\xi / H_{0}=\left\{\cdot 0306-962 \nu^{2}-\cdot 305 \nu^{4}-0102 v^{6}+\cdot 018 v^{8}-\ldots\right\} \cos (\sigma t+\epsilon)$,

Depth 29,040 feet ; $\beta=10$.

$$
\xi / H_{0}=\left\{\cdot 0298-\cdot 926 \nu^{2}-\cdot 587 \nu^{4}-\cdot 152 \nu^{6}+\cdot 029 \nu^{8}-\ldots\right\} \cos (\sigma t+\epsilon) .
$$

Depth 14,520 feet $; \beta=20$.

$$
\xi / H_{0}=\left\{0284-858 \nu^{2}-1 \cdot 08 \nu^{4}-735 \nu^{6}-056 \nu^{8}-\ldots\right\} \cos (\sigma t+\epsilon) .
$$

Depth 7,260 feet ; $\beta=40$.

$$
\xi / H_{0}=\left(0260-740 \nu^{2}-1 \cdot 62 \nu^{4}-1 \cdot 89 \nu^{6}-893 \nu^{8}-\ldots\right) \cos (\sigma t+\epsilon) .
$$

Lunar fortnightly tide. Boundary $\nu_{1}=\frac{1}{2}$, Lat. $60^{\circ}$.

Depth 58,080 feet ; $\beta=5$.

$$
\begin{aligned}
\zeta / H_{0}=\left\{\cdot 117-\cdot 856 \nu^{2}-\cdot 285 \nu^{4}-\cdot 0158 \nu^{6}+\cdot 0136 \nu^{8}+\cdot 0091 \nu^{10}+\right. & \left.\cdot 0056 \nu^{12} \ldots\right\} \\
& \times \cos (\sigma t+\epsilon) .
\end{aligned}
$$


Depth 29,040 feet ; $\beta=10$.

$$
\begin{aligned}
\xi / H_{0}=\left\{\cdot 105-\cdot 737 \nu^{2}-\cdot 493 \nu^{4}-\cdot 096 \nu^{6}+\cdot 0170 \nu^{8}+\cdot 0208 \nu^{10}+\cdot\right. & \left.0133 \nu^{12} \ldots\right\} \\
& \times \cos (\sigma t+\epsilon) .
\end{aligned}
$$

Depth 14,520 feet ; $\beta=20$.

$$
\begin{aligned}
\xi / H_{0}=\left\{0887-\cdot 557 \nu^{2}-\cdot 751 \nu^{4}-\cdot 358 \nu^{6}-\cdot 0441 \nu^{8}+\right. & 0382 \nu^{10}+ \\
& \left.\cdot 0353 \nu^{12} \ldots\right\} \\
& \times \cos (\sigma t+\epsilon) .
\end{aligned}
$$

Depth 7,260 feet ; $\beta=40$.

$\zeta / H_{0}=\left\{\cdot 0668-\cdot 333 \nu^{2}-\cdot 916 \nu^{4}-\cdot 915 \nu^{6}-\cdot 468 \nu^{8}-\cdot 0680 \nu^{10}+\cdot 0708 \nu^{12} \ldots\right\}$

$\times \cos (\sigma t+\epsilon)$.

\section{Tides of Second Species.}

The equations to be solved are (29), together with the boundary condition $u=0$ at $\nu=\nu_{1}$. From (24) the latter turns out to be

$$
0=\sum_{n=1}^{\infty} \frac{f^{2} \beta A_{n-2}-A_{n}+f^{2} \beta}{f-f^{2} n} \underline{B_{n-2}} \nu_{1}^{n},
$$

or $\quad \sum_{n=1}^{\infty} A_{n}\left\{\frac{f^{2} \beta \nu_{1}^{n+2}}{f-f^{2}(n+2)}-\frac{\nu_{1}^{n}}{f-f^{2} n}\right\}=-\Sigma \frac{f^{2} \beta B_{n}}{f-f^{2}(n+2)} \nu_{1}^{n+2}$.

The only value of $B$ occurring in the tide-producing potential is $B_{1}=H_{1}$. Hence, writing, as before,

$$
\begin{aligned}
& L_{n}=\left\{\frac{\left(f^{2}-1\right) \beta}{\frac{1}{f}-(n+2)}-\frac{n\left(n+1-\frac{1}{f}\right.}{\frac{1}{f}-n}\right) \frac{\frac{1}{f}-(n+2)}{(n+2)^{2}-1}, \\
& M_{n}=\frac{\beta}{\frac{1}{f}-(n+2)} \frac{\frac{1}{f}-(n+4)}{(n+4)^{2}-1}, \\
& a_{n}=\frac{f^{2} \beta \nu_{1}^{n+2}}{f-f^{2}(n+2)}-\frac{\nu_{1}^{n}}{f-f^{2} n} ;
\end{aligned}
$$


1913.] The dynamical theory of the tidzs in a polar basin.

the equations to be solved are

$$
\begin{aligned}
& \sum_{1}^{n} a_{n} A_{n}=-\frac{\beta H_{1} \nu_{1}^{3}}{\frac{1}{f}-3} \\
& L_{1} A_{1}+A_{3}=-\frac{\left(f^{2}-1\right) \beta H_{1}}{8} \\
& M_{1} A_{1}+L_{3} A_{3}+A_{5}=-\frac{\left(5-\frac{1}{f}\right) \beta H_{1}}{\left(3-\frac{1}{f}\right) 24} \\
& M_{n} A_{n}+L_{n+2} A_{n+2}+A_{n+4}=0
\end{aligned}
$$

and thereafter

The determinant for the free oscillations is

$$
\left|\begin{array}{cccccc}
\alpha_{1} & \alpha_{3} & \alpha_{5} & \alpha_{7} & \alpha_{9} & \ldots \\
L_{1} & 1 & 0 & 0 & 0 & \ldots \\
M_{1} & L_{3} & 1 & 0 & 0 & \ldots \\
0 & M_{3} & L_{5} & 1 & 0 & \ldots \\
0 & 0 & M_{5} & L_{7} & 1 & \ldots \\
\ldots & \ldots & \ldots & \ldots & \ldots
\end{array}\right|=0 .
$$

Approximations to the first roots of this equation are found by putting all powers of $\nu_{1}$ above the third equal to zero. Then

$$
\begin{aligned}
& \alpha_{1}=-\frac{\nu_{1}}{f-f^{2}}+\frac{f^{2} \beta \nu_{1}^{3}}{f-f^{2} \cdot 3} \\
& \alpha_{3}=-\frac{\nu_{1}^{3}}{f-f^{2} \cdot 3},
\end{aligned}
$$

the other values of $\alpha$ vanishing.

The equation to be solved is

$$
I_{41}=\frac{a_{1}}{a_{3}}
$$

or $\quad \frac{\left(f^{2}-1\right) \beta}{8}+\frac{\left(\frac{1}{f}-2\right)\left(\frac{1}{f}-3\right)}{\left(\frac{1}{f}-1\right) 8}=-\left(\frac{\beta_{\nu 1}^{2}}{\frac{1}{f}-3}-\frac{1}{f-f^{2}}\right) \div \frac{\nu_{1}^{2}}{f-3 f^{2}}$. 
This reduces to

$$
\frac{9}{8} \beta f^{4}-\frac{9}{8} \beta f^{3}-f^{2}\left(\frac{\beta}{8}+\frac{3}{4}+\frac{3}{v_{1}^{2}}\right)+f\left(\frac{\beta}{8}+\frac{5}{8}+\frac{1}{\nu_{1}^{2}}\right)-\frac{1}{8}=0 .
$$

The solution of this equation exhibits four roots: two large, one positive and one negative; and two smaller, both positive. On malking $\omega$ zero, the two smaller roots vanish and the larger roots become equal with opposite signs. This is exactly analogous to the results found by Hough.* The oscillations of long period apparently only exist when the rotation is not zero. In the other case they reduce to tidal currents.

In the accompanying table, we have only shewn the first of the smaller roots. The second may be judged to belong to the next complete set of roots. The approximate values only are given. But it is clear that, were the frequencies required for a given basin, they could be exactly evaluated by means of the formulæ given in the same manner as was done in the case of the tides of first species.

TABLE III.-Lowest frequencies (approximate) of tides of second species. Boundary $\nu_{1}=\frac{1}{4}$, Lat. $75^{\circ} 30^{\prime}$.

\begin{tabular}{|c|c|}
\hline Depths. & Frequencies $(\sigma / 2 x)$. \\
\hline $\begin{array}{c}58,080 \text { feet } \\
\beta=5\end{array}$ & $\left.\begin{array}{r}3 \cdot 35 \\
-2 \cdot 70 \\
\cdot 007\end{array}\right\}$ \\
\hline $\begin{array}{c}29,040 \text { feet } \\
\beta=10\end{array}$ & $\left.\begin{array}{c}2.51 \\
-1.89 \\
\cdot 0069\end{array}\right\}$ \\
\hline $\begin{array}{c}14,520 \text { feet } \\
\beta=20\end{array}$ & $\left.\begin{array}{c}1 \cdot 95 \\
-1.29 \\
.0065\end{array}\right\}$ \\
\hline $\begin{array}{c}7,260 \text { feet } \\
\beta=40\end{array}$ & $\left.\begin{array}{c}1 \cdot 58 \\
-\cdot 91 \\
\cdot 0058\end{array}\right\}$ \\
\hline
\end{tabular}

* Phil. Trans. (A), Yol, 191 (1898), p. 159. 
$\mathrm{T}_{\mathrm{ABLE}}$ IV.-Lowest frequencies (approximate) of tides of second species. Boundary $\nu_{1}=\frac{1}{2}$, Lat. $60^{\circ}$.

\begin{tabular}{|c|c|}
\hline Depths. & Frequencies $(\sigma / 2 \omega)$. \\
\hline $\begin{array}{c}58,080 \text { feet } \\
\beta=5\end{array}$ & $\left.\begin{array}{r}1.98 \\
-1.32 \\
.024\end{array}\right\}$ \\
\hline $\begin{array}{c}29,040 \text { feet } \\
\beta=10\end{array}$ & $\left.\begin{array}{r}1.58 \\
-\quad \cdot 93 \\
.022\end{array}\right\}$ \\
\hline $\begin{array}{c}14,520 \text { feet } \\
\beta=20\end{array}$ & $\left.\begin{array}{r}1 \cdot 34 \\
-\quad \cdot 68 \\
\cdot 018\end{array}\right\}$ \\
\hline $\begin{array}{c}7,260 \text { feet } \\
\beta=40\end{array}$ & $\left.\begin{array}{c}1 \cdot 17 \\
-\cdot 51 \\
\cdot 013\end{array}\right\}$ \\
\hline
\end{tabular}

In solving equations (42) for the values of the coefficients in the case of the forced tides, it is worthy of note that, in the case $f=\frac{1}{2}$, which corresponds to the luni-solar diurnal tide $K_{1}$, if we disregard the boundary condition, the remaining equations are satisfied by

$$
A_{9}=A_{5}=A_{7}=\ldots=0 \text {, }
$$

and

$$
A_{1}=-H_{1} \text {. }
$$

Hence

$$
\xi=\xi^{\prime}+\bar{\xi}=\nu \sqrt{ }\left(1-\nu^{2}\right)\left(-H_{1}+H_{1}\right)=0 .
$$

This is Laplace's famous result that the diurnal tide for the case of an ocean of constant depth covering the whole globe produces no rise and fall of the surface.

In the case of the other diurnal tides, $f$, while not rigorously equal to $\frac{7}{2}$, is not far from that value. Hence, as shewn by Hough, the whole of the diurnal tides are small. It becomes, therefore, of importance to see the effect of a boundary in altering these conditions. As the numerical work in any case is very complicated we shall confine our attention to the case of $f=\frac{1}{2}$. 
In (42) put $H_{1}=\kappa A_{1}$ as before. The second and third equations then become

$$
\left.\begin{array}{l}
\left(L_{1}-\frac{3}{32} \beta_{\kappa}\right) A_{1}+A_{3}=0 \\
\left(M_{0}+\frac{1}{8} \beta_{\kappa}\right) A_{1}+L_{3} A_{3}+A_{5}=0
\end{array}\right\}
$$

These equations can be solved in precisely the same way as was done in the case of the fortnightly tide. The following are the results :-

Luni-solar diurnal tide. Boundary $\nu_{1}=\frac{1}{4}$, Lat. $75^{\circ} 30^{\prime}$.

Depth 58,080 feet ; $\beta=5$.

$\xi / H_{1}=\sqrt{ }\left(1-v^{2}\right)\left\{\cdot 895 v-\cdot 518 v^{3}-\cdot 132 v^{5}-\cdot 122 v^{7} \ldots\right\} \cos (\sigma t+\phi+\epsilon)$.

Depth 29,040 feet ; $\beta=10$.

$\left.\xi / H_{1}=\sqrt{ }\left(1-\nu^{2}\right) \mid \cdot 805 \nu-1 \cdot 12 \nu^{3}-2 \cdot 76 v^{5}-2 \cdot 725 \nu^{7}-2 \cdot 348 v^{9} \ldots\right\}$

Depth 14,520 feet; $\beta=20$.

$\times \cos (\sigma t+\phi+\epsilon)$.

$\xi / H_{1}=\sqrt{ }\left(1-\nu^{2}\right)\left\{\cdot 648 \nu-2 \cdot 53 \nu^{3}-6 \cdot 34 \nu^{5}-6 \cdot 43 \nu^{7}-5 \cdot 05 \nu^{9} \ldots\right\} \cos (\sigma t+\phi+\varepsilon)$.

Depth 7,260 feet $; \beta=40$.

$\xi / H_{1}=\sqrt{ }\left(1-\nu^{2}\right)\left\{\cdot 703 \nu-4 \cdot 87 \nu^{3}-15 \cdot 7 \nu^{5}-18 \cdot 3 \nu^{7}-13 \cdot 2 \nu^{9} \ldots\right\} \cos (\sigma t+\phi+\varepsilon)$.

Luni-solar diurnal tide. Boundary $\nu_{1}=\frac{1}{2}$, Lat. $60^{\circ}$.

Depth 58,080 feet $; \beta=5$.

$\xi / H_{1}=\sqrt{ }\left(1-\nu^{2}\right)\left\{\cdot 634 \nu-\cdot 640 \nu^{3}-1 \cdot 30 \nu^{5}-1 \cdot 25 \nu^{7}-\cdot 928 \nu^{9}-\cdot 837 \nu^{4} \ldots\right\}$

Depth 29,040 feet $; \quad \beta=10$.

$\times \cos (\sigma t+\phi+\epsilon)$.

$\zeta / H_{1}=\sqrt{ }\left(1-\nu^{2}\right)\left\{\cdot 408 \nu-1 \cdot 49 \nu^{3}-2 \cdot 84 \nu^{5}-2 \cdot 69 \nu^{7}-2 \cdot 30 \nu^{9}-2 \cdot 022 \nu^{11}\right.$

Depth 14,520 feet ; $\beta=20$.

$-1 \cdot 813 \nu^{13} \ldots ; \cos (\sigma t+\phi+\epsilon)$.

$\xi / H_{1}=\sqrt{ }\left(1-\nu^{2}\right)\left\{\cdot 211 \nu-3 \cdot 36 \nu^{3}-6 \cdot 82 \nu^{5}-6 \cdot 43 \nu^{7}-4 \cdot 90 \nu^{9}-3 \cdot 86 \nu^{11}\right.$

Depth 7,260 feet ; $\beta=40$.

$\left.-3 \cdot 28 \nu^{13} \ldots\right\} \cos (\sigma t+\phi+\epsilon)$.

$\xi / H_{1}=\sqrt{ }\left(1-\nu^{2}\right)\left\{\cdot 267 \nu-6 \cdot 59 \nu^{3}-17 \cdot 6 \nu^{5}-19 \cdot 1 \nu^{7}-12 \cdot 9 \nu^{9}-7 \cdot 18 \nu^{11}\right.$

$-4 \cdot 32 \nu^{13} \ldots: \cos (\sigma t+\phi+\varepsilon)$. 
1913.] The dynamical theory of the tides in a polar basin.

\section{Tides of Third Species.}

The results for the tides of the third species are derived from the formulæ (12), (15), (16), and (17) by putting $s=2$. In the tide-producing potential the only term appearing is $B_{0}=H_{2}$. The frequencies are determined from (21) after putting $\kappa=0, s=2$. As previously, the approximate values of the first set of roots are given by

or

$$
L_{0}=\frac{1}{\nu_{1}^{2}}
$$

$$
2 \beta f^{3}-\beta f^{2}-f\left(\beta+12+\frac{12}{\nu_{1}^{2}}\right)+2=0 .
$$

The roots of this equation exhibit the same characteristics as those of the equation in the second species. There are two large roots, one positive and one negative, and one small positive root. When the rotation disappears the small root becomes zero and the others tend to numerical equality. It is noticeable also that the large roots tend to numerical equality with increasing depth. These two results are consequent upon the reduction in value of $\beta$, which is $\frac{4 \omega^{2} a^{2}}{g h}$.

TABLE V.-Lowest frequencies (approximate) of tides of third species.

\begin{tabular}{|c|c|}
\hline Deptbs. & Frequencies $(\sigma / 2 \omega)$. \\
\hline $\begin{array}{c}58,080 \text { feet } \\
\beta=5\end{array}$ & $\left.\begin{array}{c}4 \cdot 82 \\
-4 \cdot 33 \\
\cdot 0096\end{array}\right\}$ \\
\hline $\begin{array}{c}29,040 \text { feet } \\
\beta=10\end{array}$ & $\left.\begin{array}{c}3.53 \\
-3.03 \\
.0093\end{array}\right)$ \\
\hline $\begin{array}{c}14,520 \text { feet } \\
\beta=20\end{array}$ & $\left.\begin{array}{c}2 \cdot 64 \\
-2 \cdot 15 \\
\cdot 0089\end{array}\right\}$ \\
\hline $\begin{array}{c}7,260 \text { feet } \\
\beta=40\end{array}$ & $\begin{array}{c}2 \cdot 01 \\
-1.52 \\
\cdot 0082\end{array}$ \\
\hline
\end{tabular}

Boundary $\nu_{1}=\frac{1}{4}$, Lat. $75^{\circ} 30^{\prime}$. 
TABLE VI.-Lowest frequencies (approximate) of tides of third species. Boundary $\nu_{1}=\frac{1}{2}$, Lat. $60^{\circ}$.

\begin{tabular}{|cr|}
\hline Depthis. & Frequencies $(\sigma / 2 \omega)$. \\
& \\
58,080 feet & $2 \cdot 80$ \\
$\beta=5$ & $-2 \cdot 33$ \\
& $\cdot 03$ \\
& $2 \cdot 12$ \\
29,040 feet & $-1 \cdot 66$ \\
$\beta=10$ & $\cdot 029$ \\
& $1 \cdot 69$ \\
14,520 feet & $-1 \cdot 21$ \\
$\beta=20$ & $\cdot 025$ \\
& $1 \cdot 39$ \\
7,260 feet & -91 \\
$\beta=40$ & $\cdot 020$ \\
&
\end{tabular}

It is interesting to examine determinant (21) with a view to discovering the:possibility of synchronism between the free and foreed oscillations of this species. All the semi-diurnal tides have valnes of $f$ near to 1 . For the luni-solar semi-diurnal tide $\left(K_{2}\right) f$ is rigorously unity. If in (21) we put $f=1$, it becomes an equation in $\beta$, the solution of which would give the possible depths at which synchronism could take place. As a first approximation, in (45) put $f=1$. We have then $\beta=$ about 480 for $\nu_{1}=\frac{1}{2}$, and 6490 for $\nu_{1}=\frac{1}{4}$.

Using these approximate values we may, by the process of trial and error, find the exact solution of (21). We then arrive at $\beta=552$ and 7760 respectively. These values correspond to depths 526 feet for $\nu_{1}=\frac{1}{2}$, and 38 feet for $\nu_{1}=\frac{1}{4}$. The smallness of these depths precludes the possibility of synchronism in oceans of depths comparable with the average depth of the ocean as it is on the earth.

Of course, the equation (21) is satisfied by other values of $\beta$. But these will be higher than those already calculated, and will lead to smaller depths still.

The semi-diurnal tides may be calculated from the analytical results previously given by using the proper value of $f$. We append the expansions for the tide $K_{2}$, worked out in the manner suggested. 
1913.] The dynamical theory of the tides in a polak bagin.

Luni-solar semi-diurnal tide. Boundary $\nu_{1}=\frac{1}{4}$, Lat. $75^{\circ} 30^{\prime}$.

Depth 58,080 feet ; $\beta=5$.

$\xi / H_{2}=\nu^{2}\left\{1+\cdot 0522 \nu^{2}-\cdot 592 \nu^{4}-\cdot 421 \nu^{6}-\cdot 258 \nu^{8} \ldots\right\} \cos (\sigma t+2 \phi+\epsilon)$.

Depth 29,040 feet ; $\beta=10$.

$\xi / H_{2}=\nu^{2}\left\{1+\cdot 104 \nu^{2}-1 \cdot 06 \nu^{4}-\cdot 856 \nu^{6}-\cdot 477 \nu^{8} \ldots\right\} \cos (\sigma t+2 \phi+\epsilon)$.

Depth 14,520 feet ; $\beta=20$.

$\xi / H_{2}=\nu^{2}\left\{1+\cdot 209 \nu^{2}-2 \cdot 365 \nu^{4}-1 \cdot 829 \nu^{6}-\cdot 665 \nu^{8} \ldots\right\} \cos (\sigma t+2 \phi+\varepsilon)$.

Depth 7,260 feet $; \beta=40$.

$\xi / H_{2}=\nu^{2}\left\{1+\cdot 419 \nu^{2}-4 \cdot 75 v^{4}-3 \cdot 76 v^{6}-\cdot 172 \nu^{8} \ldots\right\} \cos (\sigma t+2 \phi+\epsilon)$.

Luni-solar semi-diurnal tide. Boundary $\nu_{1}=\frac{1}{2}$, Lat. $60^{\circ}$.

Depth 58,080 feet ; $\beta=5$.

$\zeta / H_{2}=\nu^{2}\left\{1+\cdot 213 \nu^{2}-\cdot 492 \nu^{4}-\cdot 371 \nu^{6}-\cdot 244 \nu^{8}-\cdot 175 \nu^{10}-\cdot 137 \nu^{12}-\cdot 111 \nu^{14} \ldots\right\}$

Depth 29,040 feet ; $\beta=10$.

$\times \cos (\sigma t+2 \phi+\epsilon)$.

$$
\begin{array}{r}
\xi / H_{2}=\nu^{2}\left\{1+\cdot 428 \nu^{2}-\cdot 982 \nu^{4}-\cdot 795 \nu^{6}-\cdot 460 \nu^{8}-\cdot 290 \nu^{10}-\cdot 207 \nu^{12}-\cdot 162 \nu^{14} \ldots\right\} \\
\times \cos (\sigma t+2 \phi+\varepsilon) .
\end{array}
$$

Depth 14,520 feet ; $\beta=20$.

$\xi / H_{2}=\nu^{2}\left\{1+869 \nu^{2}-2 \cdot 02 \nu^{4}-1 \cdot 80 \nu^{6}-\cdot 810 \nu^{8}-\cdot 314 \nu^{10}-\cdot 154 \nu^{12}-\cdot 100 \nu^{14} \ldots\right\}$

Depth 7,260 feet ; $\beta=40$.

$\times \cos (\sigma t+2 \phi+\epsilon)$.

$\zeta / H_{2}=\nu^{2}\left\{1+1 \cdot 83 \nu^{2}-3 \cdot 86 \nu^{5}-4 \cdot 53 \nu^{6}-1 \cdot 26 \nu^{8}-1 \cdot 87 \nu^{10}-1 \cdot 21 \nu^{12}-\cdot 658 \nu^{14} \ldots\right\}$

$\times \cos (\sigma t+2 \phi+\epsilon)$.

6. Conclusion.

An examination of the expressions for the tide heights in a polar basin shows that they do not differ in form from those for an ocean wholly covering the globe ; the differences of the tides are merely in magnitude. However, the following remarks seem worthy of attention :-

(i) As the table below shows, the fortnightly tide is very much reduced 
in magnitude. Indeed, it is minute when compared with the value given by the equilibrium theory. The table exhibits a comparison between the heights of the tides at the pole and at the boundary as given by this theory, as given by the theory for an ocean covering the globe completely, and as given by the equilibrium theory. The entries in columns three and six are taken, for depths giving $\beta=10$ and $\beta=40$, from Darwin's paper,* and for depth $\beta=5$ from Lamb. ${ }^{\dagger}$ Unfortunately, Lamb uses $f=0$, though this apparently makes only a small difference in the result. Hough $\ddagger$ has given the most complete expressions, but his results include the self-attraction of the water particles; and, as this has been omitted throughout the present paper, a comparison with them would be misleading.

Boundary $\nu_{1}=\frac{1}{4}$, Lat. $75^{\circ} 30^{\prime}$.

\begin{tabular}{|c|c|c|c|c|c|c|}
\hline & \multicolumn{3}{|c|}{ Tide height at Pole. } & \multicolumn{3}{c|}{ Tide height at Boundary. } \\
\cline { 2 - 7 } & $\begin{array}{c}\text { Present } \\
\text { theory. }\end{array}$ & $\begin{array}{c}\text { Theory of } \\
\text { complete globe. }\end{array}$ & $\begin{array}{c}\text { Equilibrium } \\
\text { theory. }\end{array}$ & $\begin{array}{c}\text { Present } \\
\text { theory. }\end{array}$ & $\begin{array}{c}\text { Theory of } \\
\text { complete globe. }\end{array}$ & $\begin{array}{c}\text { Equilibrium } \\
\text { theory. }\end{array}$ \\
\hline$\beta=5$ & $\cdot 0306 H_{0}$ & $\cdot 434 H_{0}$ & $\cdot 6667 H_{0}$ & $-\cdot 0307 H_{0}$ & $-\cdot 401 H_{0}$ & $\cdot 6042 H_{0}$ \\
$\beta=10$ & $\cdot 0298 H_{0}$ & $-314 H_{0}$ & $\cdot 6667 H_{0}$ & $-\cdot 0305 H_{0}$ & $-255 H_{0}$ & $\cdot 6042 H_{0}$ \\
$\beta=20$ & $\cdot 0284 H_{0}$ & -- & $\cdot 6667 H_{0}$ & $-\cdot 0293 H_{0}$ & - & $\cdot 6042 H_{0}$ \\
$\beta=40$ & $-0260 H_{0}$ & $\cdot 104 H_{0}$ & $\cdot 6667 H_{0}$ & $-\cdot 0270 H_{0}$ & $-\cdot 1023 H_{0}$ & $\cdot 6042 H_{0}$ \\
\hline
\end{tabular}

Boundary $\nu_{1}=\frac{1}{2}$, Lat. $60^{\circ}$.

\begin{tabular}{|c|c|c|c|c|c|c|}
\hline & \multicolumn{3}{|c|}{ Tide height at Fole. } & \multicolumn{3}{c|}{ Tide height at Boundary. } \\
\cline { 2 - 7 } & $\begin{array}{c}\text { Present } \\
\text { theory. }\end{array}$ & $\begin{array}{c}\text { Theory of } \\
\text { complete globe. }\end{array}$ & $\begin{array}{c}\text { Equilibrium } \\
\text { theory. }\end{array}$ & $\begin{array}{c}\text { Present } \\
\text { theory. }\end{array}$ & $\begin{array}{c}\text { Theory of } \\
\text { complete globe. }\end{array}$ & $\begin{array}{c}\text { Equilibrium } \\
\text { theory. }\end{array}$ \\
\hline$\beta=5$ & $\cdot 117 H_{0}$ & $\cdot 434 H_{0}$ & $\cdot 6667 H_{0}$ & $-\cdot 115 H_{0}$ & $-\cdot 302 H_{0}$ & $\cdot 4167 H_{0}$ \\
$\beta=10$ & $\cdot 105 H_{0}$ & $\cdot 314 H_{0}$ & $\cdot 6667 H_{0}$ & $-\cdot 112 H_{0}$ & $-\cdot 144 H_{0}$ & $\cdot 4167 H_{0}$ \\
$\beta=20$ & $\cdot 0887 H_{0}$ & - & $\cdot 6667 H_{0}$ & $-\cdot 103 H_{0}$ & - & $\cdot 4167 H_{0}$ \\
$\beta=40$ & $\cdot 0668 H_{0}$ & $\cdot 104 H_{0}$ & $\cdot 6667 H_{0}$ & $-\cdot 0899 H_{0}$ & $-\cdot 117 H_{0}$ & $\cdot 4167 H_{0}$ \\
\hline
\end{tabular}

N.B.-The quantity $H_{0}$ used here $=-H^{r}$ in Lamb, and $-E$ in Darwin.

* Proc. Roy. Soc., Vol. xuI (1886), p. 337 ; Collected Works, Vol. I, p. 370.

+ Hydrodynamics, p. 321.

$\ddagger$ Loc. cit., p. 240. 
It is to be noticed that all three theories give the tide in the same phase at the pole, but at the boundary the two dynamical theories give the opposite phase from that of the equilibrium theory.

The tide heights required by the present theory are so small compared with the other two that it seems worth while to verify the work by reference to the problem of a plane circular rotating sheet of water, which should present results not much different from those given by the smaller of the two basins just discussed.

Using the notation of Lamb, ${ }^{*}$ the equations for the case of symmetry about the axis $(s=0)$ are

$$
\frac{\partial^{2} \xi^{\prime}}{\partial r^{2}}+\frac{1}{r} \frac{\partial \xi^{\prime}}{\partial r}-\kappa^{2} \xi=0, \quad \kappa^{2}=\frac{4 \omega^{2}}{g h}
$$

and the boundary condition

$$
\frac{\partial \xi^{\prime}}{\partial r}=0 \quad \text { at } \quad r=r_{1}
$$

In the first equation we have put

$$
f^{2}=\frac{\sigma^{2}}{4 \omega^{2}}=0
$$

as a close approximation.

The tide-producing potential will be now

$$
\bar{\zeta}=H_{0}\left(\frac{2}{3}-\nu_{1}^{2} \frac{r^{2}}{r_{1}^{2}}\right)
$$

$\nu_{1}$ being the boundary taken in the spherical problem.

The solution is

$$
\frac{\xi}{H_{0}}=\frac{4 \nu_{1}^{2}}{\kappa^{2} r_{1}^{2}}-\frac{2 \nu_{1}^{2}}{\kappa r_{1} I_{1}\left(\kappa r_{1}\right)} I_{0}(\kappa r)
$$

So that the tide height at the pole is

$$
\left\{\frac{4 \nu_{1}}{\kappa^{2} r_{1}^{2}}-\frac{2 \nu_{1}^{2}}{\kappa r_{1} I_{1}\left(\kappa r_{1}\right)}\right\} H_{0}
$$

at the boundary it is $\quad\left\{\frac{4 \nu_{1}^{2}}{\kappa^{2} r_{1}^{2}}-\frac{2 \nu_{1}^{2}}{\kappa r_{1}} \frac{I_{0}\left(\kappa r_{1}\right)}{I_{1}\left(\kappa r_{1}\right)}\right\} H_{0}$ 
Remembering that

$$
\kappa^{2} r_{1}^{2}=\frac{4 \omega^{2} r_{1}^{2}}{g h}=\frac{4 \omega^{2} a^{2}}{g h} \frac{r_{1}^{2}}{a^{2}}=\beta v_{1}^{2}
$$

we can, with the aid of tables* of the functions $I_{s}(z)$, evaluate the above expressions. The results are, for $\nu_{1}=\frac{1}{4}$,

$$
\begin{array}{lcc} 
& \text { Pole. } & \text { Boundary. } \\
\beta=5, & \cdot 032 H_{0} & -\cdot 031 H_{0}, \\
\beta=20, & \cdot 029 H_{0} & -\cdot 030 H_{0} .
\end{array}
$$

These figures completely verify the results given in the previous tables and establish the extreme smallness of the fortnightly tide in such a basin as that considered. It is also worthy of notice how very far from the truth the equilibrium tide is.

(ii) The diurnal and semi-diurnal tides both vanish at the pole in agreement with the equilibrium theory. At the boundary each shows positive values if the basin is of small extent. But the diurnal tide, for larger basins, rapidly becomes largely negative. The values at the boundary are given in the following table :-

\begin{tabular}{|l|c|c|c|c|}
\hline \multirow{2}{*}{} & \multicolumn{2}{|c|}{ Diurnal tides. } & \multicolumn{2}{l|}{ Semi-diurnal tides. } \\
\cline { 2 - 5 } & $\nu_{1}=\frac{2}{4}$ & $\nu_{1}=\frac{1}{2}$ & $\nu_{1}=\frac{1}{4}$ & $\nu_{1}=\frac{1}{2}$ \\
\hline$\beta=5$ & $\cdot 212 H_{1}$ & $\cdot 167 H_{1}$ & $\cdot 0625 H_{2}$ & $\cdot 254 H_{2}$ \\
$\beta=10$ & $\cdot 178 H_{1}$ & $-084 H_{1}$ & $.0626 H_{2}$ & $.257 H_{2}$ \\
$\beta=20$ & $\cdot 114 H_{1}$ & $-.502 H_{1}$ & $\cdot 0627 H_{2}$ & $\cdot 265 H_{2}$ \\
$\beta=40$ & $.082 H_{1}$ & $-1 \cdot 24 H_{1}$ & $\cdot 0629 H_{2}$ & $\cdot 285 H_{2}$ \\
\hline
\end{tabular}

$$
\text { Equitibrium tide }\left\{\begin{array}{ccc}
\text { Diurnal ......... } & \nu_{1}=\frac{1}{4} & \nu_{1}=\frac{1}{2} \\
\text { Semi-diurnal ... } & \cdot 0625 H_{1} & \cdot 433 H_{1} \\
\cdot 25 H_{2}
\end{array}\right.
$$

It is noteworthy that the semi-diurnal tide has, in each of the cases 
worked out, a value very near that given by the equilibrium theory. In the case of the plane circular basin there is exact equality.**

When the ocean completely covers the globe the luni-solar diurnal tide vanishes entirely, and the other diurnal tides, except at an approach to synchronism with one of the free modes, are small. In the last table the numbers for the diurnal tides are large in most cases. I have therefore made a comparison of the diurnals and semi-diurnals at the various depths to ascertain their relative importance. From Darwin's tables $+H_{1} / H_{2}=4.5$ about. It is at once seen that in all cases the diurnal tide is the greater, having from $1 \frac{1}{2}$ to about 20 times the range of the semi-diurnal in the examples given. This remarkable effect of a boundary in increasing a tide from zero to such importance can readily be verified by reference again to the plane circular rotating sea.

Near the pole the tide producing potential may be written $H_{1} \nu_{1} \cdot r / r_{1}$ for the diurnal tides, and $H_{2} \nu_{1}^{2}\left(r / r_{1}\right)^{2}$ for the semi-diurnals ; where $r_{1}$ is the radius of the basin.

Using Lamb's results, + the tide elevation at the edge $\left(r=r_{1}\right)$ will be, for the diurnal tide,

$$
\frac{3 I_{1}(z)}{I_{1}(z)+z I_{0}(z)} H_{1} \nu_{1},
$$

where

$$
z=\frac{\nu_{1}}{2} \sqrt{3 \beta} .
$$

The semi-diurnal dynamical height is the same as the equilibrium beight, viz., $H_{2} \nu_{1}^{2}$.

On evaluating these quantities for the usual values of $\beta$ and $\nu_{1}=\frac{1}{4}$, we have

$$
\begin{aligned}
\frac{\text { Diurnal height }}{\text { Semi-diurnal height }} & =17 \cdot 7 \text { for } \beta=5, \\
& =17 \cdot 3 \text { for } \beta=10, \\
& =16.7 \text { for } \beta=20 . \\
& =15.6 \text { for } \beta=40 .
\end{aligned}
$$

These results verify completely those given by the general theory.

* Lamb, p. 309 (27), and p. 311 (12).

$\dagger$ Darwin, Collected Scientific Papers, Vol. I, pp. 20, 21.

$\ddagger$ Hydrodynamics, p. 309.

SER. 2. VOL. 14. NO. 1225 . 
In his discussions of the tidal observations * of the "Discovery" and "Nimrod" Antarctic Expeditions, Sir G. Darwin has drawn attention to the fact that the diurnal tides are relatively of much more importance than the semi-diurnals in high latitudes. And while it is not suggested that there is any resemblance between the Antarctic Ocean and the basins considered in this paper, yet it is clear that the exaggeration of the diurnal tides can be accounted for in a dynamical theory. The Arctic Ocean would be nearer in form to the oceans we have been considering, but the observations of the tides seem less complete. Harris gives two sets of reductions, + but in them the semi-diurnal greatly preponderates. He gives reasons $\ddagger$ for thinking, however, that the Arctic tides are derived from the Atlantic Ocean, and not generated in the limits of the Arctic Ocean itself.

* Collected Scientific Papers, Vol. I, p. 372 ; Vol. Iv, p. 473.

+ Manual of Tides, Vol. Iva, p. 664.

$\ddagger$ Ibid., Vol. rvb, p. 386 . 